\title{
AN AUTOMATIC EXTRACTION METHOD FOR THE PARAMETERS OF MULTI-LOD BIM MODELS FOR TYPICAL COMPONENTS OF WOODEN ARCHITECTURAL HERITAGE
}

\author{
Haoyu Liu ${ }^{1,3,4}$, Miaole Hou ${ }^{1,3,4}$, Aiqun $\mathrm{Li}^{1,2,3}$, Linlin $\mathrm{Xie}^{2, *}$ \\ ${ }^{1}$ Beijing Advanced Innovation Center for Future Urban Design, Beijing University of Civil Engineering and Architecture, Beijing \\ 100044, China - 2108521517041@stu.bucea.edu.cn (H.L.), houmiaole@bucea.edu.cn (M.H.), liaiqun@bucea.edu.cn (A.L.), \\ xielinlin@bucea.edu.cn(L.X.) \\ ${ }^{2}$ School of Civil and Transportation Engineering, Beijing University of Civil Engineering and Architecture, Beijing 100044, China \\ ${ }^{3}$ School of Geomatics and Urban Spatial Informatics, Beijing University of Civil Engineering and Architecture, Beijing 100044, \\ China \\ ${ }^{4}$ Beijing Key Laboratory for Architectural Heritage Fine Reconstruction \& Health Monitoring, Beijing 100044, China
}

\section{Commission II, WG II/8}

KEY WORDS: Wooden architectural, Architectural heritage protection, BIM model, Automatic extraction method, Point cloud processing

\begin{abstract}
:
A demand-oriented Building Information Model (BIM) model built using high-fidelity point cloud data can better protect architectural heritage. The multi-level detail (mutli-LoD) parametric model emphasizes the different protection requirements of typical components and the automatic extraction of corresponding parameters of high-fidelity point clouds, which are two related key issues. Taking the typical Chinese wooden architectural heritage as an example, according to different requirements, the multi-LoD principle of typical components is proposed. On this basis, the automatic extraction method of the above parameters is developed, and the key parameters of the method are recommended. In order to solve the above problems, taking the three typical Dou-Gong used in Liao Dynasty and Song Dynasty, including Zhutou Puzuo, Bujian Puzuo and Zhuanjiao Puzuo, as an example, briefly introduced the standardization characteristics of the typical components of the "Yingzao Fashi". Subsequently, the corresponding multiple LoD principles are recommended according to different requirements. Based on this and high-fidelity point cloud data, an automatic extraction method for multi-LoD BIM model parameters for typical components of wooden architectural heritage is proposed.
\end{abstract}

\section{INTRODUCTION}

As a typical architectural heritage worldwide, wooden architectural heritage (referred to as "WAH" hereafter) is considered with long history, various architectural styles and mature systems, which can significantly reflect the cultural characteristics and advanced levels of construction technology at different historical stages (Chun et al., 2017). Hence, the WAH is usually regarded with high historical, cultural and scientific values. Among these, due to magnificent appearance and high level of construction technology, Chinese WAH is world-renowned with extremely high values, such as Yingxian Wood Pagoda which was constructed in 1506 with a height of $65.88 \mathrm{~m}$ and is the oldest and tallest ancient wooden building in China (Zhang, 2007) and (Hou et al., 2016). Scientific conservation of such heritage has become a critical issue.

3D digital technology has become an important method to support the conservation of WAH, whether it is well preserved or has a certain degree of degradation (Balletti et al., 2014). This is attributed to the fact that such technology can document the high-fidelity information. 3D digital model established by this technology is required to be capable of illustrating the characteristic construction method, historical and cultural values more intuitively.
As for the multi-LoD, the component model with different levels of details are provided in BIM, and are selected according to the importance and rendering difficulties (Clark, 1976). Furthermore, different requirements for the parametric model may exist in different conservation phase. Hence, aiming to different conservation requirements, a multi-LoD modeling system is firstly required. Subsequently, in conjunction with the concept of multi-LoD, a multi-LoD parametric model should be implemented in BIM (Murphy et al., 2009) and (Pocobelli, 2018). With this model, 3D BIM model can be established with a high efficiency, and modified/updated to adapt to the continuously evolution of WAH (Azhar et al., 2012) and (Murphy et al., 2013) and (Worrell, 2015). Therefore, an appropriate multi-LoD parametric model for the typical components of Chinese WAH and a high-efficiency automatic extraction method for the parameters of multi-LoD BIM models is necessary (Dore et al., 2015) and (Barazzetti et al., 2015).

To address the abovementioned issues, taking the Chinese WAH constructed in Liao Dynasty and Song Dynasty as an example, the standardized characteristics of typical components following "Yingzao Fashi" are briefly introduced. Subsequently, the correspondingly multi-LoD principles are recommended according to different level of requirements. The multi-LoD parametric models are conclusively recommended for these typical components. Based on this and high-fidelity point cloud data, an automatic extraction method for the parameters of

\footnotetext{
* Corresponding author E-mail addresses: xielinlin@bucea.edu.cn(Linlin Xie)
} 
multi-LoD BIM models for typical components of WAH is proposed.

\section{MULTI-LOD PARAMETRIC MODEL FOR DOU- GONG AND ITS TYPICAL COMPONENTS}

\subsection{Multi-LoD Parametric Model Principles}

Note that the beams and columns in WAH can be represented using the multi-LoD parametric models existed in BIM, while the Dou-Gong composed by various special components, as the most distinctive component in Chinese WAH, requires a new multi-LoD parametric model. It is composed by "Dou", "Gong", "Ang" and "Fang" as shown in Figure 1 with special configuration.

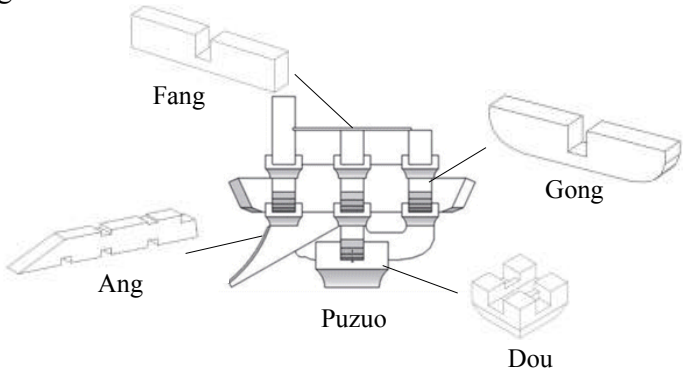

Figure 1. Schematic diagram of a Dou-Gong.

In comparison with the components in modern wooden structures, the multi-LoD principles should be developed with the consideration of the commonality and characteristic. Based on this requirement, three LoD is proposed for Dou-Gong and typical components according to different level of requirements, the principles are presented as follows:

(1) LoD100: this LoD reflects the volume information of DouGong and typical components for basic requirements of BIM model, including the length, width and height of the outer bounding box, which strictly follows the existed principle in BIM.

(2) LoD200: this LoD concentrates on the requirements of information for structural safety performance assessment, the dimensions of connection details between components and other critical dimensions are essential for such assessment and therefore are mostly concerned.

(3) LoD300: this LoD aims at the requirements of high-fidelity representation of Dou-Gong and typical components, which can demonstrate the inherent architectural culture and enable the safety performance based on high-fidelity refined model. Hence, the curved outline is implemented.

Based on the abovementioned principles, the schematic diagram of multi-LoD models are illustrated in Figure 2.

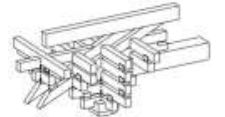

(b)
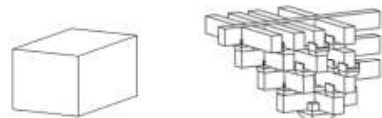

(d)

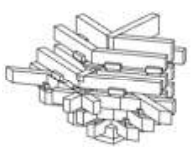

(c)

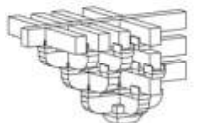

(e)

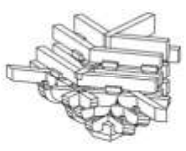

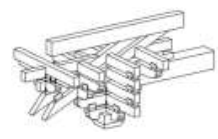

Figure 2. Multi-LoD models for Dou-Gong: (a) LoD100; (b) LoD200 for Zhutou Puzuo; (c) LoD300 for Zhutou Puzuo; (d) LoD200 for Bujian Puzuo; (e) LoD300 for Bujian Puzuo; (f) LoD200 for Zhuanjiao Puzuo; (g) LoD300 for Zhuanjiao Puzuo.

Due to the length limitation, only the schematic diagram of four representatively typical components, including one Dou, Gong, Ang and Fang, are presented in Table 1. Note that the LoD200 and LoD300 models of entire Dou-Gong are composed by the LoD200 and LoD300 models of typical components.

Name LoD100 LoD200 LoD300

Table 1. Multi-LoD models for typical components.

\subsection{Multi-LoD Parametric Model for Typical Components}

The overall diagram and disassembly diagram of three selected Dou-Gong are presented in Figure 3. It is worth mentioning here that the configuration and name of each component are also presented in Figure 3. The typical components in these Dou-Gong are summarized and categorized into 27 types. 


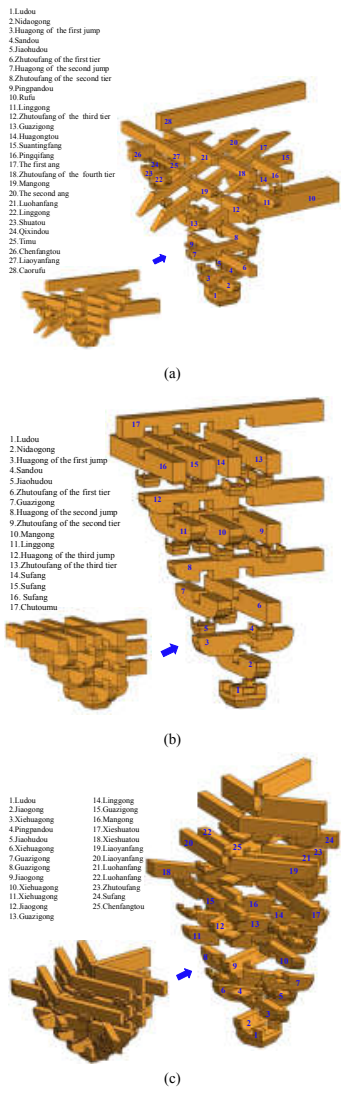

Figure 3. Overall diagram and disassembly diagram of three selected Dou-Gong; (a) Zhutou Puzuo; (b) Bujian Puzuo; (c) Zhuanjiao Puzuo.

The corresponding name and configuration are presented in Table 2.

\begin{tabular}{|c|c|c|c|}
\hline Name & $\begin{array}{c}\text { Schematic } \\
\text { diagram }\end{array}$ & Name & $\begin{array}{c}\text { Schematic } \\
\text { diagram }\end{array}$ \\
\hline Ludou & & Guazi & \\
\hline Sandou & & Mangong & \\
\hline Jiaohudou & & Jiaogong & \\
\hline Qixindou & & Ang & \\
\hline Pingpandou & & Sufang & \\
\hline Nidaogong & & $\begin{array}{l}\text { Zhutou } \\
\text { fang }\end{array}$ & \\
\hline Huagong & & $\begin{array}{c}\text { Suanting } \\
\text { fang }\end{array}$ & \\
\hline Linggong & & $\begin{array}{l}\text { Pingqi } \\
\text { fang }\end{array}$ & \\
\hline Xiehuagong & & $\begin{array}{l}\text { Luohan } \\
\text { fang }\end{array}$ & \\
\hline Liaoyanfang & & Caorufu & \\
\hline
\end{tabular}

\begin{tabular}{cccc}
\hline Name & $\begin{array}{c}\text { Schematic } \\
\text { diagram }\end{array}$ & Name & $\begin{array}{c}\text { Schematic } \\
\text { diagram }\end{array}$ \\
\hline Shuatou & $\begin{array}{c}\text { Chutoum } \\
\text { Chenfang } \\
\text { tou }\end{array}$ & Rufu \\
Huagongtou
\end{tabular}

Table 2. Typical components in three types of Dou-Gong.

\section{AN AUTOMATIC EXTRACTION METHOD FOR THE PARAMETERS OF MULTI-LOD BIM MODELS BASED ON POINT CLOUD DATA}

Specifically, the point cloud data obtained by 3D laser scanning technology is usually enormous. If the characteristic parameters of multi-LoD models can be extract from the point cloud data, the abovementioned parametric model with dozens parameters will be more convenient and practical for the establishment of BIM models. Due to these facts, an automatic extraction method is required for the parameters of multi-LoD BIM models based on point cloud data.

It is notable that the extraction method for a real WAH or even a Dou-Gong is still an extremely challenging task. Hence, this research mostly focuses on the development of an automatic extraction method at the component level, and it is expected to be further developed to meet the real engineering requirements. In the following section, the framework of the proposed method is firstly introduced step by step. Subsequently, the algorithm of each step and the associated key parameters are introduced in detail.

\subsection{Framework of the proposed method}

The overall framework of the proposed method is schematically illustrated in Figure 4. A brief introduction of the overall framework is presented step by step with emphasis on the fundamental concept of each step as follows:

(1) Step 1: Volume to slice. A point cloud slicing algorithm is adopted to transform the scatted volumetric points to layered slice, which aims to obtain the typically cross-sectional characteristics of key components. Specifically, a series of flaky point cloud is extracted from the volumetric point cloud, which will be beneficial to increase the accuracy and efficiency of the following outline extraction.

(2) Step 2: Slice to outline. An outline extraction algorithm is applied to the flaky point cloud to extract contour features, which represents the contour of key components. The outline point cloud can lay a foundation for further extraction of characteristic points.

(3) Step 3: Outline to characteristic points. A characteristic point detection algorithm is adopted to detect the characteristic points, which will be used to calculate the characteristic parameters of multi-LoD BIM models. This algorithm basically relies on the geometric relationship between the characteristic points of the outline.

(4) Step 4: Characteristic points to parameters. An extraction algorithm for the characteristic parameters of multi-LoD models is proposed to calculate the dimensions of key components. It is notable that the parameters required for LoD100, LoD200 and 
LoD300 are different. Hence, this algorithm is designed with three levels to extract the parameters of three LoD.

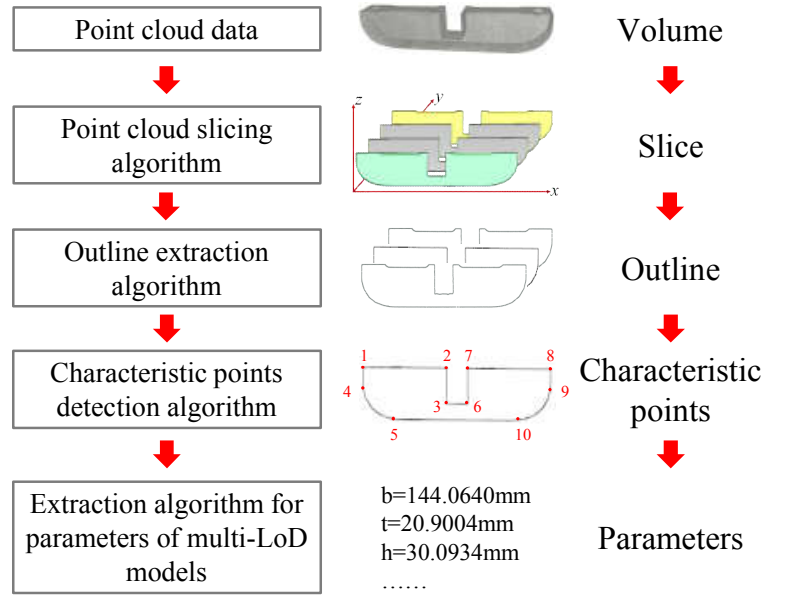

Figure 4. Overall framework.

\subsection{Point cloud slicing algorithm}

It is well acknowledged that the point cloud data is obtained with high sampling density and the corresponding quality can be affected by the environment and acquisition system. It can be found from Table 1 that each components exhibits typically cross-sectional characteristics. Hence, the introduction of slicing algorithm can accurately reflect such characteristics, thus effectively improving the efficiencies of outline extraction and characteristic point detection. Specifically, one reference axis normal to the slicing plane (e.g. Y axis as shown in Figure 6) is firstly selected according to characteristics of specific components. Then, the correspondingly maximum (i.e. $y_{\max }$ ) and minimum (i.e. $y_{\text {min }}$ ) values of the coordinate of all point cloud along the reference axis are obtained to determine the slicing spacing (i.e. $s$ ) using the following equation:

$$
s=\frac{\left|y_{\max }-y_{\min }\right|}{N}
$$

where $\quad N=$ the number of flaky point cloud sliced from the volumetric point cloud

$s=$ slicing spacing

Subsequently, $\mathrm{N}$ minus one projection planes with an identical spacing (i.e. $s$ ) and $\mathrm{N}$ groups of point cloud data are generated as shown in Figure 5.
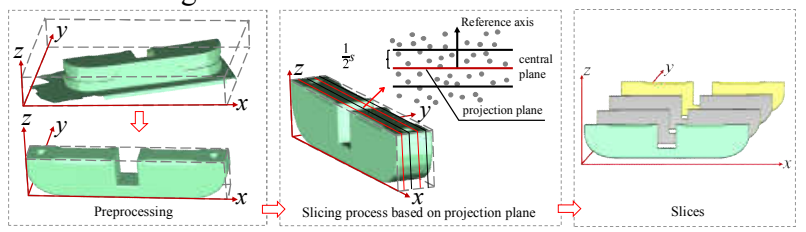

Figure 5. Schematic diagram of the slicing algorithm based on point cloud.

\subsection{Outline extraction algorithm}

Enormous data remains in the flaky point cloud sliced from the volumetric point cloud, the outline containing the characteristic points is required to be further extracted.

An outline extraction algorithm based on point cloud clustering is adopted here, in which the point cloud data is firstly reorganized using the K-D tree method (Steder et al., 2011). Subsequently, point cloud clustering is performed before the calculation of distribution density. Hence, the points on the outline remain while the interference points are eliminated. Due to these, the accuracy and efficiency of outline extraction can be significantly improved. The fundamental procedures of this algorithm are schematically presented in Figure 6 and demonstrated as follows:

a) An index relationship is established using the K-D tree method and the point cloud clustering is conducted.

b) The normal vector of each point is calculated and used to construct least square plane.

c) All points after point cloud clustering are traversed. If the maximum angle difference is greater than the specified threshold, the point belongs to the outline, otherwise it is identified as the internal node. The points on the outline are conclusively identified and stored as a new object, i.e. the outline.

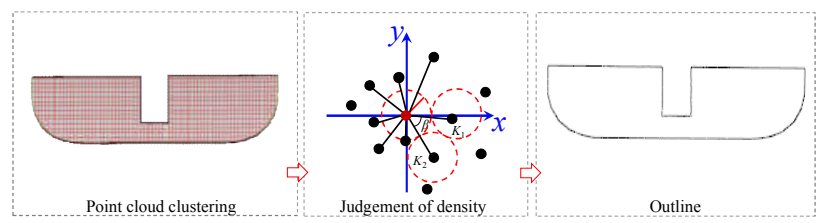

Figure 6. Schematic diagram of the outline extraction algorithm.

\subsection{Characteristic point detection algorithm}

To obtain the parameters of multi-LoD models, the characteristic points are usually corner points on the outline and required to be further identified, indicating that a characteristic point detection algorithm should be introduced. A 3D Harris method is expanded from traditional 2D Harris method and adopted to extract the characteristic points (i.e. interest points usually referred). This is attributed to the fact that this method is robust to the change of attitude of object and is basically immune to the ration (Lv et al., 2013).

In addition, a series of tests indicate that some interfering points may be identified during the process, which should be eliminated for the extraction of characteristic parameters. Two types of such points are usually observed, including the intermediate points on an expected linear outline (i.e. type 1 as shown in Figure 7) and the points on the curved outline (i.e. type 2 as shown in Figure 7).

For type 1, this interfering point is extracted because an ideally linear outline cannot be perfectly manufactured, and can be directly eliminated. To achieve this, the angle between two direction vectors of three continuous points are calculated. If this angle is more than the threshold (e.g. the angle between vector 41 and vector 16 as shown in Figure 7), the middle point (e.g. 1 in Figure 7) of three continuous points (e.g. 4, 1 and 6 in Figure 7) is a characteristic point and is herein reserved. In contrast, the middle point (e.g. point 6 in Figure 7) is identified as the interfering point and eliminated.

For type 2, the points should be further processed to obtain two characteristic points and the key parameter $\mathrm{m}$. As mentioned above, $\mathrm{m}$ minus one inflection points exist on such curved outline. The angle between two direction vectors of three continuous points will significantly change $\mathrm{m}$ plus one times, including two characteristic points (e.g. point 4 and 5 in Figure 7) and m minus one inflection points (e.g. point 7,8 and 9 in Figure 7). According to this characteristic, these points can be further extracted and $\mathrm{m}$ can be calculated. Subsequently, the 
starting and ending points are reserved as characteristic points, while the intermediate points are eliminated.

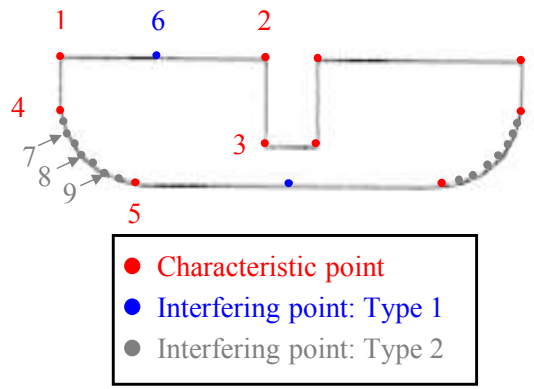

Figure 7. Schematic diagram of the characteristic points and interfering points.

The fundamental procedures of this algorithm are schematically presented in Figure 8. The search radius (denoted as "sr") of fixed window in Figure 8 is the critical parameter of this algorithm.

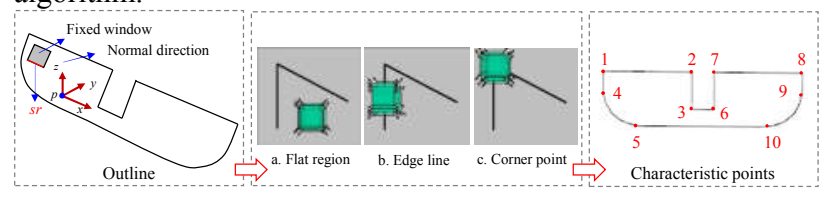

Figure 8 . Schematic diagram of the characteristic point detection algorithm.

\subsection{Extraction algorithm for the characteristic parameters} of multi-LoD models

Based on the characteristic points, the characteristic parameters of multi-LoD models can be determined according to the geometric relationship. The extraction algorithm is designed with three levels to extract the parameters of LoD100, LoD200 and LoD300, respectively.

a) Parameters of LoD100 model

The parameters of LoD100 demonstrates the volumetric information of components, including the length (i.e. $b$ ), width (i.e. $t$ ) and height (i.e. $h$ ) of the outer bounding box. An introspective sorting algorithm is adopted to obtain the maximum and minimum coordinate values in each direction (Musser, 2010). The corresponding parameters are calculated according to Equation 2:

$$
\begin{aligned}
& b=y_{\max }-y_{\min } \\
& t=x_{\max }-x_{\min } \\
& h=z_{\max }-z_{\min }
\end{aligned}
$$

\section{where $\quad b=$ length of components \\ $t=$ width of components \\ $h=$ height of components}

b) Parameters of LoD200 model

LoD200 concentrates on the requirements of information for structural safety performance assessment and simple linear outline is considered. The associate parameters are calculated according to the characteristic points. There exists two situations, the original outline is linear or curved, which has been identified in the characteristic point detection algorithm.

For the first one, the corresponding parameters are mostly internal connection dimension (e.g. $b_{1}$ and $h_{1}$ as shown in Figure $9 \mathrm{~b}$ ). These parameters are the distance between associated characteristic points, which are obtained from the characteristic point detection algorithm.

For the second one, the curved outline is simplified to a right angle as shown in Figure 9. Due to this, the associated region has not been modified in comparison with that of LoD100 model, no parameter is required to be introduced.

c) Parameters of LoD300 model

LoD300 demonstrates the inherent architectural culture and enable the safety performance based on high-fidelity refined model. The curved outline is mostly concerned and required to be represented. Basically, the curved outline is a polyline following the rules specified in "Yingzao Fashion". The parameters required to be extracted are the length (i.e. $b_{2}$ ) and height (i.e. $h_{2}$ ) of "Juansha" as shown in Figure 9c. Taking $h 2$ as an example, it is $h$ in LoD100 minus the distance between two characteristic points (i.e. point 1 and 4 as shown in Figure 7).

In addition, $\mathrm{m}$ minus one calculated inflection points, in which $\mathrm{m}$ denotes the number of segments of "Juansha" obtained in characteristic point detection algorithm, are required to be determined, thus facilitating the representation of shape grammar. To achieve this, a local coordinate system as shown in Figure is Figure9c is adopted. The function of each linear segment of polyline $\left(y_{i}\right)$ are established using Equation 3. Then, the $j^{\text {th }}$ calculated inflection point can be calculated as the intersection of $y_{j}$ and $y_{j+1}$.

$$
y_{i}=(m-i+1) h 2\left(\frac{x}{i b_{2}}+\frac{1}{m}\right) \quad i=1,2, \cdots, m
$$

where $\quad m=$ number of "Juansha"

$b_{2}=$ length of "Juansha"

$h_{2}=$ height of "Juansha"

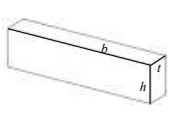

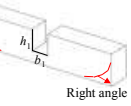

(b)

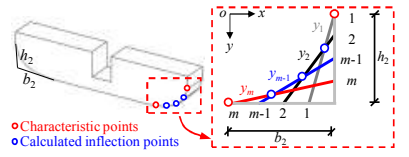

Figure 9. Schematic diagram of parameters of multi-LoD models: (a) LoD100; (b) LoD200; (c) LoD300.

\section{AN AUTOMATIC EXTRACTION METHOD FOR THE PARAMETERS OF MULTI-LOD BIM MODELS BASED ON POINT CLOUD DATA}

The information of computer used are presented in Table 3. And the point cloud of validation object is shown in Figure 10.

\begin{tabular}{cc}
\hline Information & Parameter \\
\hline Processor & Intel(R) Xeon(R) CPU E5-1603 \\
Memory & v3 \\
Operating system & 64 bit Windows7 \\
3rd Liberay & PCL 1.7.2 \\
\hline
\end{tabular}

Table 3. Information of the used computer. 


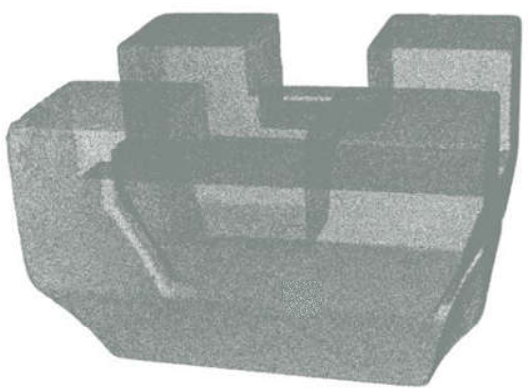

Figure 10. Point cloud of validation object.

To validate the reliability and efficiency of the proposed method, the values of parameters of multi-LoD models for Ludou are also extracted, presented and compared with the reference values in Table 4 and 5 . The absolute error and correspondingly relative error of each parameter of multi-LoD model are mostly lower than $1 \mathrm{~mm}$ and $2 \%$, respectively. Only a notable relative error with a value $3.15 \%$ is observed for a local small dimension, which is considered acceptable. The wall time is 374.137 s. A good accuracy and high efficiency are also achieved. Furthermore, the LoD300 BIM model is also established and the three-dimensional deviation analysis is also conducted, as shown in Figure 11. The correspondingly maximum deviation, average deviation, standard deviation and RMS estimate are presented in Table 8 . The deviation is overall small even negligible. The results of other component can be found in reference (Shi et al., 2018), the relative and absolute errors of parameters of multi-LoD models are all less than 3.5\% and $1 \mathrm{~mm}$, respectively. In addition, the wall times are all less than 7 minutes.

Based on the above discussion, the reliability and efficiency of the proposed method are validated. The feasibility and reliability of the proposed parametric model and automatic extraction method are validated.

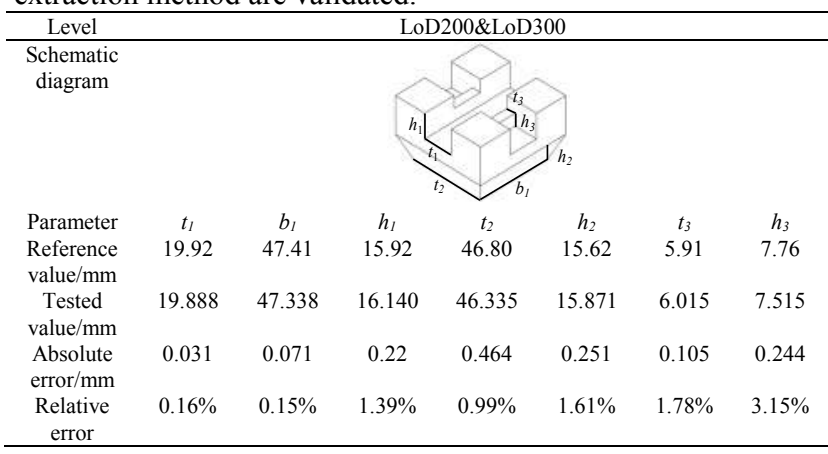

Table 4. Extraction results and accuracy of LoD200\&LoD300 of Ludou.

\begin{tabular}{cccc}
\hline Level & \multicolumn{3}{c}{ LoD100 } \\
\hline $\begin{array}{c}\text { Schematic } \\
\text { diagram }\end{array}$ & $t$ & 64.08 & 39.86 \\
$\begin{array}{c}\text { Parameter } \\
\text { Reference } \\
\text { value/mm }\end{array}$ & 63.59 & 64.939 & 40.336 \\
$\begin{array}{c}\text { Tested } \\
\text { value/mm } \\
\text { Absolute } \\
\text { error/mm } \\
\text { Relative } \\
\text { error }\end{array}$ & 64.098 & 0.859 & 0.476 \\
\hline
\end{tabular}

Table 5. Extraction results and accuracy of LoD100 of Ludou.

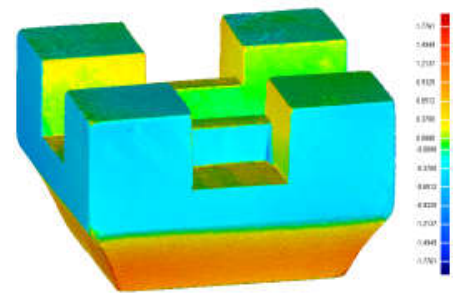

Figure 11. Results of three-dimensional deviation analysis for Ludou.

\section{CONCLUSIONS}

A framework of multi-LoD parametric models for typical components, as well as an automatic extraction method for the corresponding parameters from high-fidelity point cloud, are two critical issues in the continuous protection of WAH using BIM.

Taking the Chinese WAH constructed in Liao Dynasty and Song Dynasty as an example, the multi-LoD principles are recommended according to different level of requirements are proposed for such typical components. Based on these, the framework of multi-LoD parametric models are conclusively recommended for these typical components and implemented in BIM.

An automatic extraction method for the parameters of multiLoD BIM models for typical components is proposed. To validate the reliability and high efficiency of this method, the parameters of multi-LoD models of Ludou are extracted using the proposed method. The results indicate that the relative and absolute errors of such parameters are less than $3.5 \%$ and $1 \mathrm{~mm}$, respectively. In addition, this method is capable of extracting parameters from tens of millions of point cloud data within 7 minutes, thus validating the high efficiency and reliability of the proposed method.

The research outcomes will provide important reference for the historical data digitalization of wooden architectural heritages.

\section{REFERENCES}

Azhar, S.; Khalfan, M.; Maqsood, T., 2012. Building Information Modelling (BIM): now and beyond. Australas $J$ Constr Econ Build, 2012, 12, pp. 15-16. DOI: 10.5130/ajceb.v12i4.3032.

Balletti, C.; Berto, M.; Gottardi, C.; Guerra, F., 2014. 3D technologies for the digital documentation of an ancient wooden structure. International Journal of Heritage in the Digital Era, 2014, 3, pp. 19-32. DOI: 10.1260/20474970.3.1.19.

Barazzetti, L.; Banfi, F.; Brumana, R.; Gusmeroli, G.; Previtali, M.; Schiantarelli, G., 2015. Cloud-to-BIM-to-FEM: structural simulation with accurate historic BIM from laser scans. Simul Model Pract Theory 2015, 57, pp. 71-87. DOI: 10.1016/j.simpat.2015.06.004.

Chun, Q.; Pan, J.W.; Dong Y.H., 2017. Research on integrity damage indexes of the ancient timber frame buildings in the south China. Sciences of Conservation \& Archaeology 2017, 29, pp. 76-83. DOI: 10.16334/j.cnki.cn31-1652/k.2017.06.010. 
Clark, J.H., 1976. Hierarchical geometric models for visiblesurface algorithms. Acm Siggraph Computer Graphics 1976, 19, pp. 267-268. DOI: $10.1145 / 563274.563323$.

Dore C, Murphy M, McCarthy S, Brechin F, Casidy C, Dirix E., 2015. Structural simulations and conservation analysishistoric building information model (HBIM). ISPRS Int Arch Photogramm Remote Sens Spat Inf 2015, 40, pp. 351-357. DOI: 10.5194/isprsarchives-XL-5-W4-351-2015.

Hou, W.D.; Wang, L.A.; Yong, X.Q., 2016. Research on the protection of Yingxian Wood Tower, 1st ed.; Cultural Relics Press: Beijing, China, 2016; pp. 39-107.

Lv, J.G.; Yang, D.H.; Wang Q.S., 2013. Fast 3-D feature point detector based on Harris. Applied Mechanics and Materials 2013, 325-326, 1567-1570. DOI: 0.4028/www.scientific.net/amm.325-326.1567.

Murphy, M.; McGovern, E.; Pavia, S., 2013. Historic Building Information Modelling - adding intelligence to laser and image based surveys of European classical architecture. ISPRS Journal of Photogrammetry and Remote Sensing 2013, 76, pp. 89-102. DOI: 10.1016/j.isprsjprs.2012.11.006.

Murphy, M.; McGovern, E.; Pavia, S., 2009. Historic building information modelling (HBIM). Struct Survey 2009, 27, pp. 311-327. DOI: 10.1108/02630800910985108.

Musser, D.R., 2010. Introspective sorting and selection algorithms. Software Practice \& Experience, 2010, 27, pp. 983993. DOI: 10.1002/(sici)1097-024x(199708)27:8<983::aidspe117>3.0.co;2-\#.

Pocobelli, D.P.; Boehm, J.; Bryan, P.; Still, J.; Grau-Bové, J., 2018. BIM for heritage science: a review. Heritage Science 2018, 6, 30. DOI: 10.1186/s40494-018-0191-4.

Shi, L.W.; Hou, M.L.; Xie, L.L.; Jiang, Y.H., 2018. Research on high-efficiency dimension retention technology for ancient wooden architectural components for different accuracy requirements. Geomatics World, 2018, 25(131), pp. 1-7.

Steder, B.; Rusu, R.B.; Konolige, K.; Burgard, W., 2011. Point feature extraction on 3D range scans taking into account object boundaries. IEEE International Conference on Robotics and Automation, Shanghai, China, 2011. DOI: 10.1109/ICRA.2011.5980187.

Worrell, L.L., 2015. Building information modeling (BIM): the untapped potential for preservation documentation and management. Master's Thesis, Clemson University, American, 2015.

Zhang, J.L., 2007. The record and analysis of damage of Yingxian Wooden Tower. Master's Thesis, Taiyuan University of Technology, China, 2007. 